EGU21-10716

https://doi.org/10.5194/egusphere-egu21-10716

EGU General Assembly 2021

(c) Author(s) 2022. This work is distributed under

the Creative Commons Attribution 4.0 License.

\title{
Influence of Tropical Waves on the Lifecycle of Mesoscale Convective Systems over West Africa
}

\author{
Marlon Maranan ${ }^{1}$, Andreas Schlueter ${ }^{2}$, Andreas H. Fink ${ }^{1}$, and Peter Knippertz ${ }^{1}$ \\ ${ }^{1}$ Karlsruhe Institute of Technology, Karlsruhe, Germany (marlon.maranan@kit.edu) \\ ${ }^{2}$ Stanford University, Stanford, United States of America
}

Rainfall variability over West Africa remains a major challenge for numerical weather prediction (NWP). Due to the largely stochastic and sub-grid nature of tropical convection, current NWP models still fail to provide reliable precipitation forecasts - even for a 1-day leadtime - and are barely more skillful than climatology-based forecasts. Thus, several recent studies have investigated the presumably more predictable influence of tropical waves on environmental conditions for convection and found distinct and coherent (thermo-)dynamical patterns depending on the type and phase of the wave. Of particular interest in this context is the interaction of the wave with the lifecycle of usually westward propagating mesoscale convective systems (MCSs), which are the major providers of rain in the region and can occasionally even lead to flooding. The exact mechanisms and strength of this interaction are still not entirely known.

This study combines two recent datasets in a novel way in order to systematically investigate the influence of tropical waves on MCS characteristics and lifecycle. First, MCSs are tracked within northern tropical Africa $\left(20^{\circ} \mathrm{W}-30^{\circ} \mathrm{E} / 2^{\circ}-15^{\circ} \mathrm{N}\right)$ over an 11 -year period during the West African rainy season (April-October) using infrared brightness temperature fields provided by the Spinning enhanced visible and infrared imager (SEVIRI). Second, tropical waves are isolated by applying a filtering method in the wave-frequency domain to precipitation data of the Tropical Rainfall Measuring Mission (TRMM) within the $5^{\circ}-15^{\circ} \mathrm{N}$ latitude band for the same target period. By combining the two datasets in space and time, the magnitude and phase of each wave is known at every timestep of the MCS tracks, which enables a systematic investigation of MCS characteristics as a function of wave properties.

Preliminary results suggest that long-lived MCSs (lifetime $\geq 12 \mathrm{~h}$ ) frequently couple with the "wet" phase of high-frequency tropical waves, in particular Kelvin, eastward inertia-gravity (EIG), and African easterly waves (AEW). Showing an enhanced occurrence frequency of MCS initiation, the wet phase of AEWs appears to have strong modulation capabilities during the genesis stage and further accompanies these long-lived MCSs during their entire lifetime. In the case of Kelvin waves and EIGs, the wet phase overlaps only with the intensification and maturity stage of these MCSs as a consequence of opposite directions of movement. Similar coupling patterns also exist for mixed Rossby gravity waves (MRGs), although to a weaker extent. Furthermore, no consistent coupling tendencies with long-lived MCSs are evident for low-frequency waves (Madden-Julian Oscillation 
(MJO), equatorial Rossby wave (ER)), arguably since they act on larger spatio-temporal scales. For short-lived MCSs (lifetime < 6h), the coupling with high-frequency waves is substantially weaker.

In the future we will also address potential influences of wave-wave interactions on MCSs as well as potential differences in coupling mechanisms between the Guinea Coast region and the Sahel farther north. With increasing efforts in the prediction of tropical waves, this study has the potential to aid the short-term forecasting of MCS development and its lifecycle. This can be of particular importance for the anticipation of extreme rainfall events and subsequent risk assessment in West Africa. 